\title{
CBIR with Various Feature Extraction Techniques using LIRS and LSRR Performance Parameter
}

\author{
H.B.Kekre, Ph.D \\ Senior Professor, \\ Computer Engineering \\ Department, MPSTME, \\ SVKM's NMIMS (Deemed-to- \\ be University), Mumbai, India
}

\author{
Aditi Mehta \\ M.Tech Student, \\ Computer Engineering \\ Department, MPSTME, \\ SVKM's NMIMS (Deemed-to- \\ be University), Mumbai, India
}

\author{
Paulami shah \\ Assistant Professor, \\ Computer Engineering \\ Department, MPSTME, \\ SVKM's NMIMS (Deemed-to- \\ be University), Mumbai, India
}

\begin{abstract}
In fields such as medical, art galleries, museums, archaeology, medical imaging, trademark databases, criminal investigations, images especially the digital images grow in quantities of thousands and sometimes even lakhs every year. Content based image retrieval is required from such large databases. This paper compares various CBIR techniques based on the performance evaluation parameters namely, precision, recall, LIRS and LSRR. Euclidean Distance is used for the purpose of similarity measure.
\end{abstract}

\section{Keywords}

Content Based Image Retrieval(CBIR); Discrete Cosine Transform (DCT); Discrete Sine Transform (DST); Walsh Transform; Row Mean(RM); Column Mean(CM); Row Column Mean (RCM); Forward Diagonal Mean (FDM); Backward Diagonal Mean (BDM); Forward Backward Diagonal Mean (FBDM);Euclidean distance; Precision; Recall; Length of Initial Relevant String of images(LIRS).

\section{INTRODUCTION}

The very large numbers of images are being generated from a variety of sources (digital camera, digital video, scanner, the internet etc.) which have posed technical challenges to computer systems to store/transmit and index/manage image data effectively to make such collections easily accessible. Image compression deals with the challenge of storage and transmission, where significant advancements have been made $[1,4,5]$. The challenge to image indexing is studied in the context of image database $[2,6,7]$, which has become one of the promising and important research area for researchers from a wide range of disciplines like computer vision, image processing, image database and recognition systems.

The thirst of better and faster image retrieval techniques is increasing day by day. The ambiguity in text based retrieval emphasizes the need of a better and faster retrieval system. That is why CBIR becomes more important. Some of important applications for CBIR technology could be identified as art galleries, museums, archaeology [3], architecture design $[8,13]$, geographic information systems [5], weather forecast [5], medical imaging [5], trademark databases, criminal investigations, image search on the Internet.

\section{CONTENT BASED IMAGE RETRIEVAL}

In literature the term content based image retrieval (CBIR) has been used for the first time by Kato et.al. [4], to describe his experiments into automatic retrieval of images from a database by colour and shape feature. The typical CBIR system performs two major tasks. The first one is extraction of feature vector which consists of various feature components. It is generated to represent the content of each image in the database with accuracy and uniqueness. The second task is similarity measurement (SM), where a distance between the feature vector of the query image and the feature vector of each image in the database is measured, compared and this is used to retrieve the top "closest" images.

For feature extraction in CBIR there are mainly two approaches [5] feature extraction in spatial domain and feature extraction in transform domain. The feature extraction in spatial domain includes the CBIR techniques based on histograms [5], BTC [1,2]. The transform domain methods are widely used to extract image features. Many current CBIR systems use Euclidean distance [1-3] on the extracted feature set as a similarity measure. The Direct Euclidian Distance between image $\mathrm{P}$ and query image $\mathrm{Q}$ can be given as equation 1 , where Vpi and Vqi are the feature vectors of image $\mathrm{P}$ and Query image Q respectively with size ' $n$ '.

$$
E D=\sqrt{\sum_{i=1}^{n}\left(V_{p i}-V_{q i}\right)_{2}}
$$




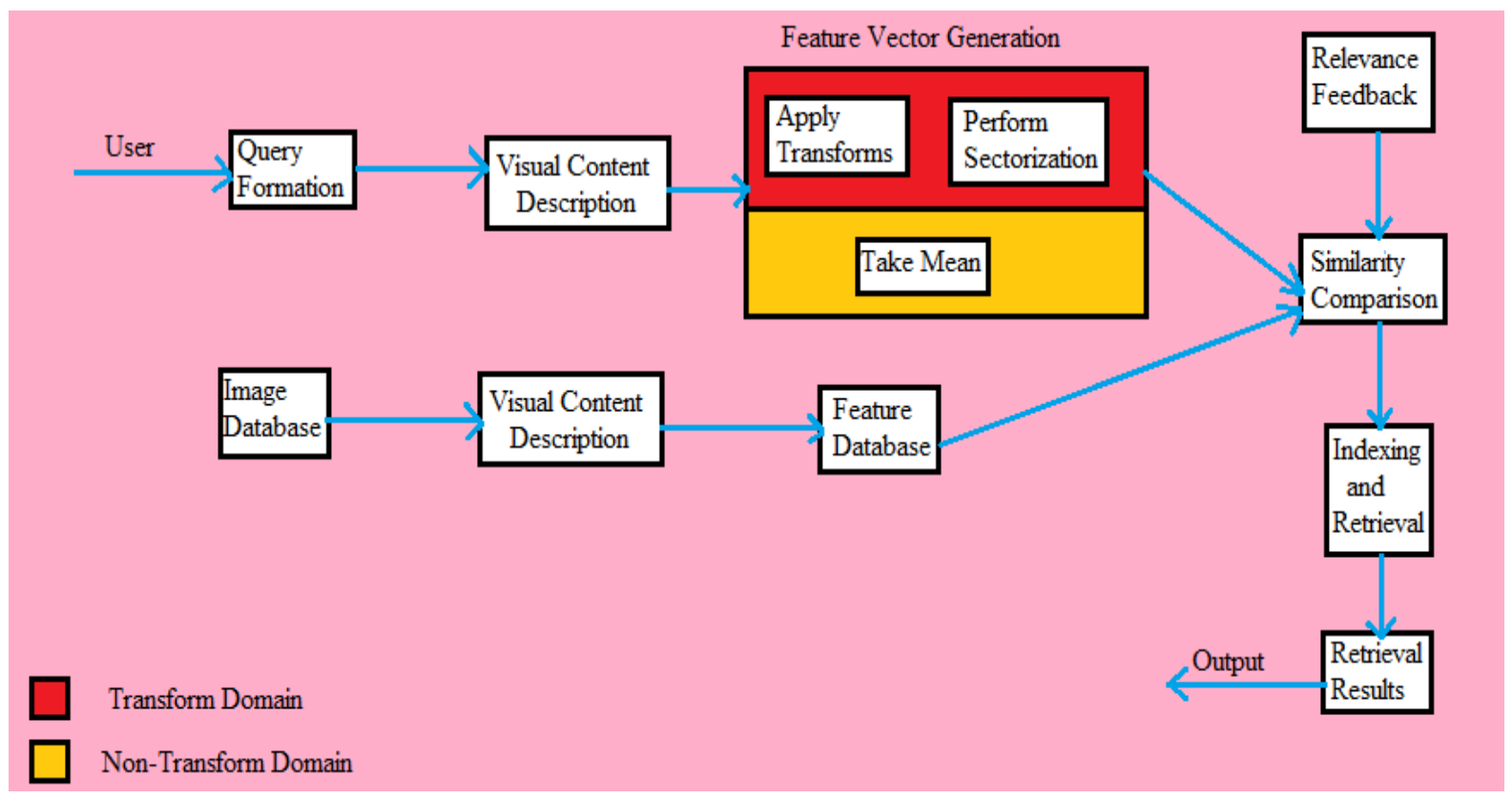

Figure 1: CBIR System [14]

In a typical content-based image retrieval system as shown in figure above, the visual contents of the images in the database are extracted and described by feature vectors. The feature vectors of the images in the database form a feature database. To retrieve images, users provide the retrieval system with example images. The system then changes these examples into its internal representation of feature vectors. The similarities /distances between the feature vectors of the query example and those of the images in the database are then calculated and retrieval is performed. This provides an efficient way to search for the image database. Recent retrieval systems have also began with taking the feedback from users for making further improvements in the retrieval results.

\section{COMPARISON OF TECHNIQUES}

Various CBIR systems were studied and all of these systems discuss different techniques of feature vector generation. All of these techniques are discussed briefly in this section.

\subsection{Row Mean of Image (RM) [11]}

In this method, Feature Vector is generated by calculating row mean of all pixels in the row of image matrix for all three planes i.e. R,G,B. Euclidean distance is used as the similarity measure and the performance is evaluated using precision and recall.

\subsection{Column Mean of Image (CM) [11]}

In this method, Feature Vector is generated by calculating column mean of all pixels in the column of image matrix for all three planes i.e. R,G,B. Euclidean distance is used as the similarity measure and the performance is evaluated using precision and recall.

\subsection{Row \& Column Mean of Image (RCM) [11]}

In this method, Feature Vector is generated by calculating row and column mean of all pixels in the column of image matrix for all three planes i.e. R,G,B. Euclidean distance is used as the similarity measure and the performance is evaluated using precision and recall.

\subsection{Backward Diagonal Mean of Image (BDM) [11]}

In this method, Feature Vector is generated by calculating backward diagonal mean of all pixels in the column of image matrix for all three planes i.e. R,G,B. Euclidean distance is used as the similarity measure and the performance is evaluated using precision and recall.

\subsection{Forward Diagonal Mean of Image (FDM) [11]}

In this method, Feature Vector is generated by calculating forward diagonal mean of all pixels in the column of image matrix for all three planes i.e. R,G,B. Euclidean distance is used as the similarity measure and the performance is evaluated using precision and recall.

\subsection{Forward \& Backward Diagonal Mean of Image (FBDM) [11]}

In this method, Feature Vector is generated by calculating forward and backward diagonal mean of all pixels in the column of image matrix for all three planes i.e. R,G,B. Euclidean distance is used as the similarity measure and the performance is evaluated using precision and recall.

\subsection{CBIR-BTC [1,2]}

In this method, Feature Vector is generated by using three independent $\mathrm{R}, \mathrm{G}$ and $\mathrm{B}$ components of color images to calculate three different thresholds and then apply BTC to each individual $\mathrm{R}, \mathrm{G}$ and $\mathrm{B}$ planes. Rest is same as mentioned in section above. Euclidean distance is used as the similarity measure and the performance is evaluated using precision and recall.

\subsection{Flipping Techniques [1,2]}

A flipped image is a static or moving image that is generated by a mirror reversal of an original across a horizontal (X) axis or vertical (Y) axis. While flipping along the $\mathrm{X}$-axis; the pixel values of the images are interchanged wherein the 1st element of the 1st row is interchanged with the last element of the $1 \mathrm{st}$ row, the $2^{\text {nd }}$ element of the 1 st row is interchanged with the 
second last element of the 1st row and so on till all the pixel values of all the rows are interchanged. Similarly, is done column wise when flipping the image along $\mathrm{Y}$ axis.

Even image $=$ Original Image + Flipped Image

Odd Image $=$ Original Image - Flipped Image

\section{i. Even BTC}

In this method, the feature vectors are generated by using the 6 feature vectors consisting of only the even part.Euclidean distance is used as the similarity measure and the performance is evaluated using precision and recall.

\section{ii. Odd BTC}

In this method, the feature vectors are generated by using the 6 feature vectors consisting of only the odd part.Euclidean distance is used as the similarity measure and the performance is evaluated using precision and recall.

iii. Even and Simple BTC

In this method, the feature vectors are generated by using a combination of both the even part as well as the simple BTC thus increasing the feature vector to size 12. Euclidean distance is used as the similarity measure and the performance is evaluated using precision and recall.

\section{iv. Odd and Simple BTC}

In this method, the feature vectors are generated by using a combination of both the odd part as well as the simple BTC thus increasing the feature vector to size 12. Euclidean distance is used as the similarity measure and the performance is evaluated using precision and recall.

\section{v. Even and Odd BTC}

In this method, the feature vectors are generated by using a combination of both the even part as well as the odd BTC thus increasing the feature vector to size 12. Euclidean distance is used as the similarity measure and the performance is evaluated using precision and recall.

vi. Even, Odd and Simple BTC

In this method, the feature vectors are generated by using a combination of the even, odd as well as the simple BTC thus increasing the feature vector to size 18. Euclidean distance is used as the similarity measure and the performance is evaluated using precision and recall.

\subsection{Row Mean of Transformed Column Image [13]}

This technique of CBIR can be explained using following steps:
1. Apply transform $\mathrm{T}$ on the column of image of size $\mathrm{NxN}$ (INxN) to get column transformed image of the same size $(\mathrm{cINxN})$

$\operatorname{cINxN}($ column transformed $)=[\mathrm{TNxN}][\mathrm{INxN}]$

2. Calculate row mean of column transformed image to get feature vector of size $\mathrm{N}$ (instead of $\mathrm{N} 2$ )

3. Then Euclidean Distance is applied to obtain precision and recall.

\section{IMPLEMENTATION}

\subsection{Database}

The CBIR techniques (3.1-3.9) are tested on a single image database, i.e. Generic Image Database which contains 1000 fixed size images spread across 10 categories of human being, Flowers, Tribal, Elephant, Scenery, animals, natural scenery and other manmade things. There are 100 images for each category. [11-13]

\subsection{Performance Evaluation Parameters}

To assess the retrieval effectiveness, precision, recall, and LIRS are used as statistical comparison parameters for the proposed CBIR techniques. The standard definitions for these measures are given by following equations.

Precision $\quad \frac{\text { Wumbey of Relewant Amages Aatyieved }}{\text { Total Wwmbey of Images Aatyieved }}$

Recall

Nwmbey of Relevant Imageg Retrieved $=$ Total Wumbey of Relewant Inages in Databaze (4)

LIRS

Length of Initial Aelevant Stying of imageg $=$ Total Wumber of Relevant Images in Databage (5)

LSRR Length of atying to recover ail images

$=$ Total Nmages in Databage

\subsection{Results}

Figure 2 compares techniques (3.1-3.11) discussed in previous sections. It compares various CBIR techniques. It is seen that CM followed by RCM and FBDM give the best results inturn followed by RM- DCT ,DST and Walsh. 


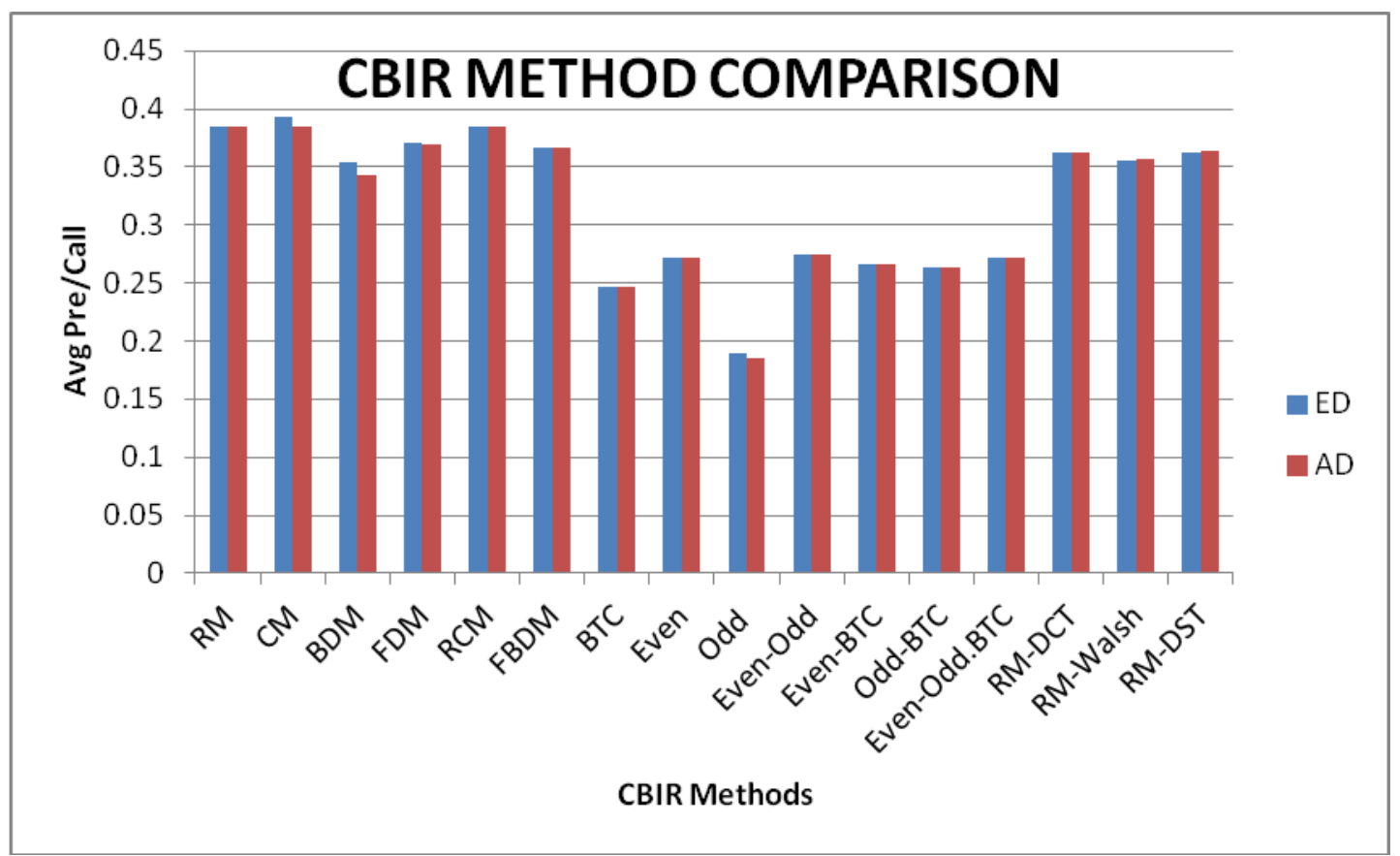

Figure 2: Graph of CBIR techniques (3.1-3.9) with respective precision \& recall crossover values.

Figure 3 compares the LIRS values of the techniques (3.13.9). It is seen that best value of LIRS is given by CM, RCM followed by FBDM and CM inturn followed by RM-DST, DCT and Walsh.

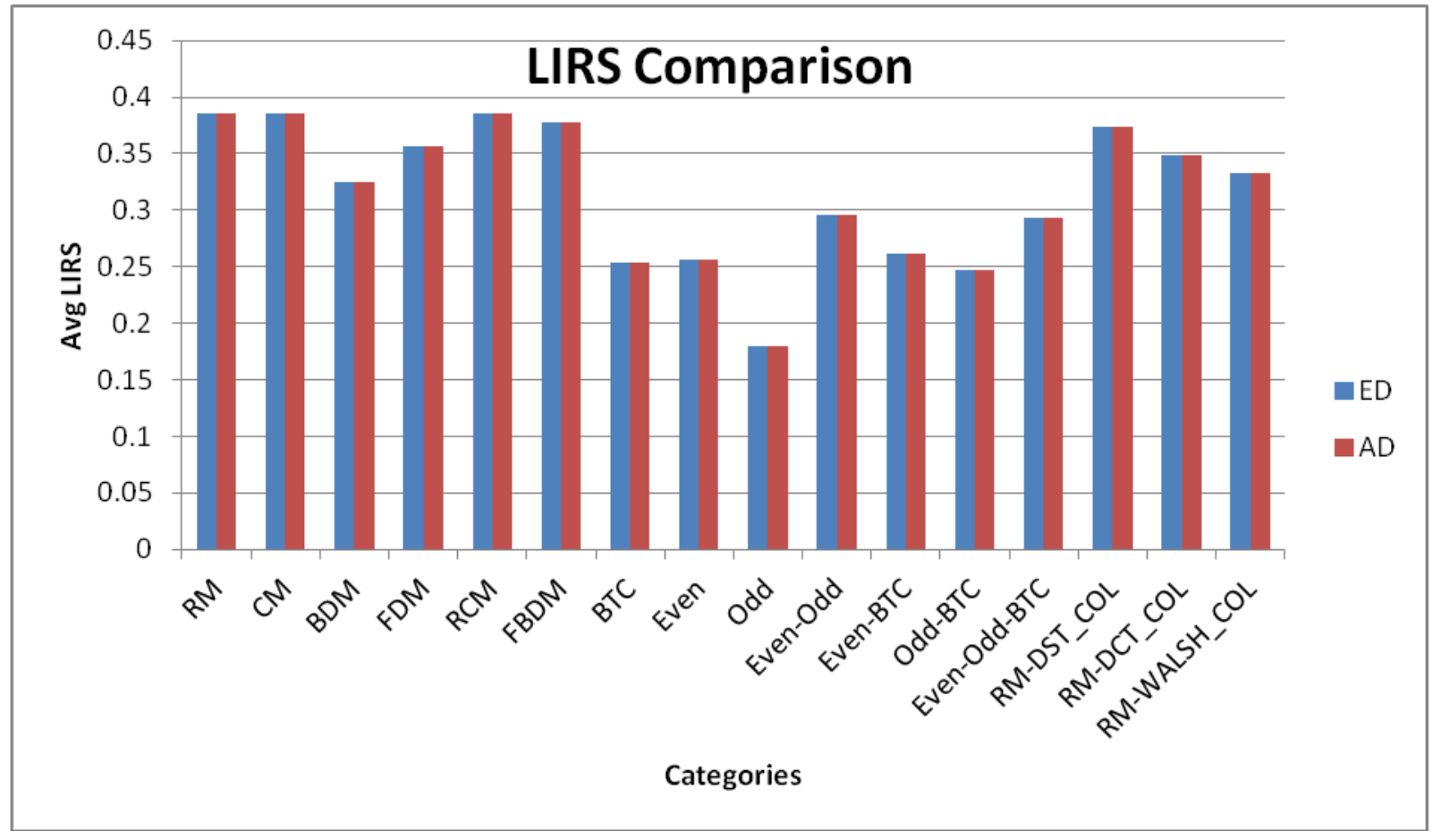

Figure 3: Graph of CBIR techniques (3.1-3.9) with respective LIRS Values

Figure 4 compares the LSRR values of the techniques (3.13.9). It is seen that best value of LSRR is given by RMDST_COL, RCM followed by FBDM and CM. 


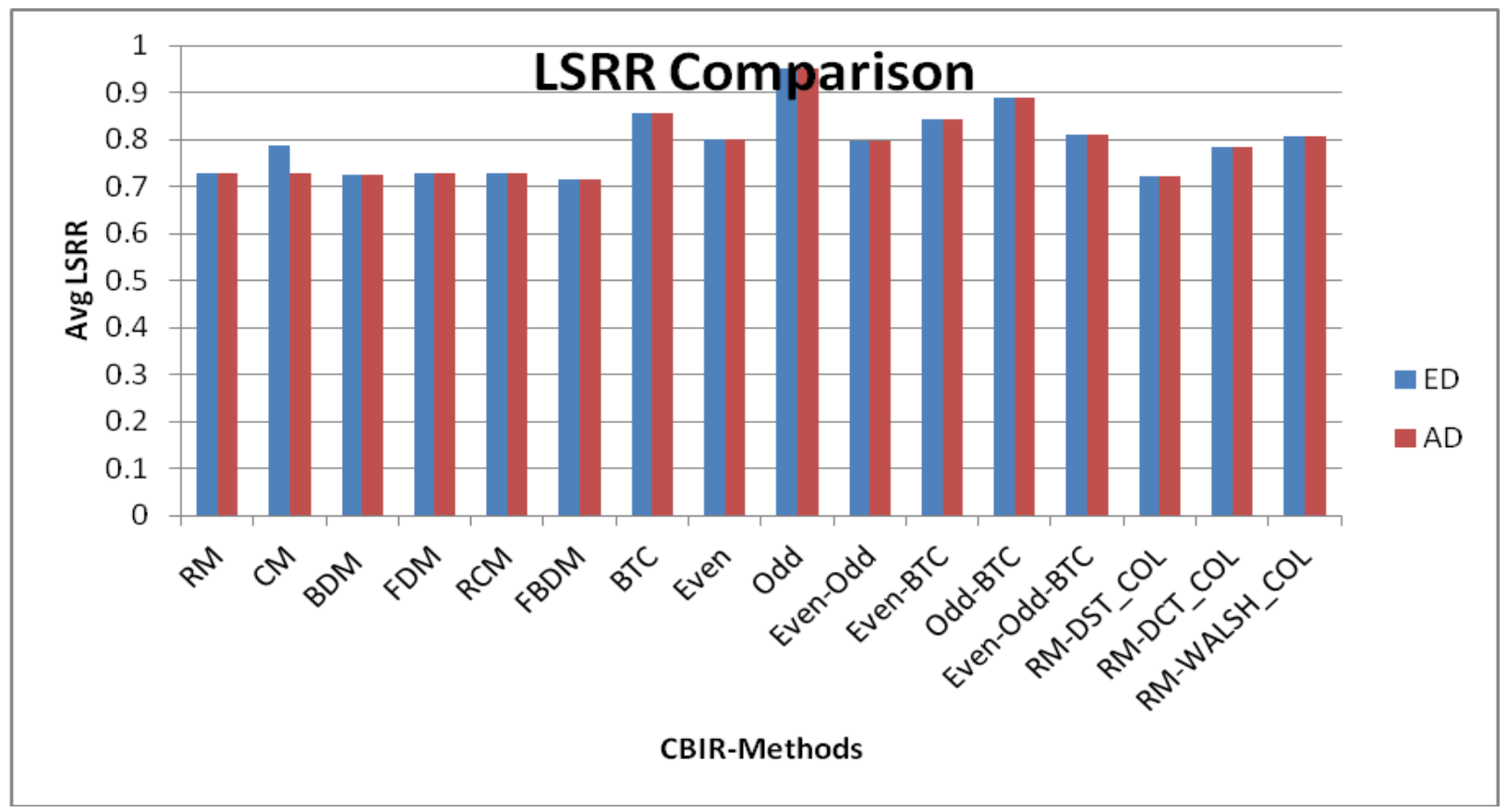

Figure 4: Graph of CBIR techniques (3.1-3.9) with respective LSRR Values

\section{CONCLUSION}

Study says that higher the value of LIRS and lower the value of LSRR, better is the performance of the CBIR method. And, from the graphs above (figure 2 and figure 3), a remarkable co-relation can be found between Precision/Recall, LIRS and LSRR i.e. Methods with a high crossover value of Precision/Recall also have a high value of LIRS and corresponding low LSRR value; and those with low crossover values of Precision/Recall have respective low values of LIRS and high values of LSRR, i.e. CM and RCM have the highest precision/recall and respective highest LIRS value and lowest LSRR values as well.

\section{ACKNOWLEDGMENTS}

I would like to thank my mentor Mrs. Paulami Shah, and Dr. H. B. Kekre, for their constant support and motivation.

\section{REFERENCES}

[1] H.B.Kekre, Sudeep D. Thepade, "Boosting Block Truncation CodingusingKekre's LUV Color Space for Image Retrieval", WASETInternational Journal of Electrical, Computer and System Engineering (IJECSE), Volume 2, Number 3, pp. 172-180, Summer 2008.

[2] H.B.Kekre, Sudeep D. Thepade, "Image Retrieval using AugmentedBlock Truncation Coding Techniques", ACM International Conferenceon Advances in Computing, Communication and Control (ICAC3-2009), pp. 384390, 23-24 Jan 2009, Fr. ConceicaoRodrigousCollegeofEngg., Mumbai. Is uploaded on online ACM portal.

[3] H.B.Kekre, Sudeep D. Thepade, "Scaling Invariant Fusion of ImagePieces in Panorama Making and Novel Image Blending Technique", International Journal on Imaging (IJI), www.ceser.res.in/iji.html,Volume 1, No. A08, pp. 31-46, Autumn 2008.

[4] Hirata K. and Kato T. "Query by visual example content-based image retrieval", In Proc. of Third
International Conference on $\mathrm{H}$ Extending Database Technology, EDBT'92, 1992, pp 56-71

[5] H.B.Kekre, Sudeep D. Thepade, "Rendering Futuristic Image RetrievalSystem", National Conference on Enhancements in Computer,Communication and Information Technology, EC2IT-2009, 20-21 Mar2009, K.J.Somaiya College of Engineering, Vidyavihar, Mumbai-77

[6] Minh N. Do, Martin Vetterli, "Wavelet-Based Texture Retrieval Using Generalized Gaussian Density and Kullback-Leibler Distance", IEEE Transactions On Image Processing, Volume 11, Number 2, pp.146158, February 2002.

[7] B.G.Prasad, K.K. Biswas, and S. K. Gupta, "Region based image retrieval using integrated color, shape, and location index", International Journal on Computer Vision and Image Understanding Special Issue: Colour for Image Indexing and Retrieval, Volume 94, Issues 1-3, April- June 2004, pp.193-233.

[8] Dr.H.B.Kekre, Dr.Dhirendra Mishra, “ DCT Sectorization for Feature Vector Generation in CBIR",International Journal of Computer Applications (IJCA) Vol.9(1) November 2010, ISSN 0975-8887 available online http://www.ijcaonline.org/volume9/number1/pxc387182 0.pdf

[9] Dr.H.B.Kekre, Dr.Dhirendra Mishra, "DCT-DST Plane sectorization of Row-wise Transformed color Images in CBIR",International Journal of Engineering Science and Technology (IJEST) Vol.2(12) 2010, ISSN 72347244available online at http://nmims.edu/wpcontent/uploads/2012/p3/MPSTME/Direndra,DCTDSTPlanesectorization.pdf

[10] Dr.H.B.Kekre, Dhirendra Mishra, “ Density distribution in WalshTransform sectors as feature vectors for image retrieval", published in international journal of compute 
applications (IJCA) Vol.4(6) 2010, 30-36 ISSN 09758887 available online at http://www.ijcaonline.org/archives/volume4/number6/82 9-1072

[11] Dr.H.B.Kekre, SudeepDThepade, AkshayMaloo, "Query by Image Content Using Colour Averaging Techniques", International Journal of Engineering Science and Technology (IJEST), Volume 2, Issue 6, 2010.pp.16121622 (ISSN: 0975-5462) Available online at http://www.ijest.info

[12] Dr.H.B.Kekre, SudeepDThepade, AkshayMaloo, "Image Retrieval using Fractional Coefficients of Transformed Image using DCT and Walsh Transform", International Journal of Engineering Science and Technology (IJEST),
Volume 2, Issue 4, 2010.pp.362-371 (ISSN: 0975-5462) Available online at http://www.ijest.info

[13] Dr.H.B.Kekre, SudeepDThepade, AkshayMaloo, "Performance Comparison of Image Retrieval using Row Mean of Transformed Column Image", International Journal of Engineering Science and Technology (IJEST), Volume 2, Issue 5, 2010.pp.1908-1912 (ISSN: 09753397) Available online at http://www.ijest.info

[14] Aditi Mehta, Paulami Shah "Review and Comparison of Various Feature Extraction Techniques in CBIR", international journal of compute applications (IJCA), Volume 71(23), ISSN 0975-8887 